\title{
The impact of follicle-flushing during oocyte collection on embryo development of in-vitro fertilization
}

\author{
Li-hua Zhu' ${ }^{\dagger}$ Xiao-bei $\mathrm{Ni}^{\dagger}$, Fei Lin, Zhi-peng Xu, Jun-shun Fang ${ }^{*}$ and Ning-yuan Zhang ${ }^{*}$
}

\begin{abstract}
Background: To evaluate the impact of follicle-flushing during oocyte collection on embryo development potential retrospectively.

Methods: A total of 1714 cases, including 133 who experienced retrieval difficulty (repeated follicle-flushing) on the day of oocyte retrieval (difficulty group) and the control 1581 cases (control group), were assessed in this retrospective study. The number of oocytes recovered, two pro-nuclei fertilization (2PN-fertilization), day 3 goodquality embryo and day 5/6 blastocyst utilization rates were compared between the difficulty group and control group correspondingly. Embryo implantation, clinical pregnancy and neonatal outcomes were further analyzed between the two groups in the fresh day ${ }^{-3}$ embryo transfer cycles.

Results: The number of oocytes recovered in the difficulty group $(9.08 \pm 4.65)$ were significantly reduced compared with the control group $(12.13 \pm 5.27), P<0.001$; The $2 \mathrm{PN}$-fertilization, day 3 good-quality embryo and blastocyst utilization rates were significantly lower in the difficulty group compared with controls $(71.7 \%$ vs. $75.7 \%$; $52.7 \%$ vs. 56.5\%; 31.9\% vs. 37.0\%, all $P<0.05$ ). Embryo implantation in the difficulty group was $53.2 \%$, which was lower than the control value of $58.7 \%$, although not reaching statistical significance. The rate of fresh embryo transfer cycles in the difficulty group was lower than normal ones $(51.88 \%$ vs. $61.99 \%, P=0.026)$. The pregnancy and live birth rates were similar between the two groups. But the rate of spontaneous miscarriages of the difficulty group was higher than the control group, although not reaching statistical significance. The neonatal outcomes had no statistical difference between the two groups.
\end{abstract}

Conclusions: Oocyte retrieval difficulty, which include repeated flushing and the corresponded extending time required for oocyte recovery, significantly reduced day 3 good-quality embryo and blastocyst utilization rates of these patients. But the live birth rate had no difference between the difficulty group and the normal ones.

Keywords: Oocyte retrieval difficulty, Number of recovered oocytes, Embryo development potential, Blastulation

\section{Background}

Transvaginal ultrasound-guided oocyte retrieval is a technique in which under ultrasound guidance, a needle is inserted through the posterior fornix to the ovary, and a mature ovum is retrieved for in-vitro fertilization and embryo culture $[1,2]$. This method has become a routine procedure for oocyte retrieval in human assisted reproduction, and is considered as an effective, safe, and

\footnotetext{
*Correspondence: fangjunshun@163.com; katty_ny@163.com

${ }^{+}$Li-hua Zhu and Xiao-bei Ni contributed equally to this work.

Reproductive Medical Center, Drum Tower Hospital Affiliated with Nanjing University Medical College, Zhongshan Road 321\#, 210008 Nanjing, People's Republic of China
}

required step for in-vitro fertilization and embryo transplantation (IVF-ET) [3-5]. It affects oocyte recovery number and oocytes quality, as well as the outcome of embryos growth $[3,6]$. On the day of oocyte retrieval, we have encountered cases that no oocytes were recovered, regardless of many matured follicles; and cases that obtained a scant number of oocytes from numerous matured follicles. These patients may experience aspiration difficulty, even facing a situation that the operative time is too long and most of their follicles are flushed repeatedly [7]. High flushing pressure might cause early rupture of the follicular wall, which results in oocytes 
damage. In this retrospective study, 133 patients who experienced retrieval difficulty on the day of oocyte collection were assessed. The oocyte development potential, the clinical and the neonatal outcomes were analyzed in the article.

\section{Methods}

\section{Study design}

This retrospective study was approved by the Medical Ethics Committee of Drum Tower Hospital Affiliated with Nanjing University Medical College. All patients provided signed informed consent for IVF-ET treatment in 2017.

This retrospective study included women with regular menses who were undergoing their first cycle of in vitro fertilization (IVF) or intracytoplasmic sperm injection (ICSI) due to tubal factors, male factors, or both. Tubal factors included unilateral or bilateral tubal occlusion, peritubal adhesion, unilateral or bilateral salpingectomy, or tubal ligation. Male-factor infertility included oligospermia, asthenospermia, or obstructive azoospermia. Eligible women were 22 to 35 years old, had a normal menstrual cycle (defined as a spontaneous cycle length of 21 to 35 days), and a duration of infertility of more than 1 year. Women with a history of unilateral oophorectomy, recurrent spontaneous abortion, diagnosis of the polycystic ovary syndrome, or uterine abnormality (e.g., adenomyosis, mullerian duct anomaly, endometriosis, submucous myoma, intra- uterine adhesion, or scarred uterus) were excluded. Women were also excluded if they had renal disease, abnormal renal function, history of deep venous thrombosis, severe anemia, pulmonary embolus, or cerebrovascular accident. All the couples were screened with the use of karyotyping, and those with an abnormal karyotype were excluded.

\section{Methods}

Each follicle with a diameter of $>10 \mathrm{~mm}$ was aspirated. The tip of the needle was directed to the centre of the follicle and the aspirate was considered complete when the follicle appeared to have completely collapsed on the ultrasound scan. This initial aspirate was handed to the embryologist, if an oocyte was not found, the dead space in the collecting system (needle and attached tubing) was then flushed with $2 \mathrm{ml}$ culture medium until the first drop of medium was seen. If an oocyte was also not found, the follicle was flushed up to a maximum of six times. All of the recovered oocytes were flushed at least twice of a patient was considered oocyte retrieval difficulty $[4,7,8]$.

This study involved 133 patients who presented to our center for the first oocyte retrieval cycle, and experienced retrieval difficulty on the day of oocyte aspiration. The control group included 1581 patients who had successful oocyte retrieval in our center. Age, Body-mass index, amounts of oocytes recovered, embryo development potential and the clinical outcome were compared between the two groups. Furthermore, based on different fertilization methods, patients receiving IVF and ICSI were individually compared for oocytes recovered, embryo development potential, and the clinical outcomes.

Ovulation induction and fertilization: Controlled ovarian hyperstimulation $(\mathrm{COH})$ was performed according to the standard protocols of our center. All patients underwent $\mathrm{COH}$ with exogenous gonadotrophins used the following protocol: long GnRH agonist protocol, in which the agonist was started in the mid luteal phase of the preceding menstrual cycle, adding gonadotropins on the second day after menstrual bleeding. And ovulation was triggered when follicles reached $>17 \mathrm{~mm}$ in diameter, using $250 \mu \mathrm{g}$ of human chorionic gonadotropin (hCG) (Ovitrelle ${ }^{\circ}$, Merck Serono, Italy). On the day of operation, routine IVF or ICSI was performed based on sperm quality.

Embryo culture: For IVF, insemination was performed after $4-5 \mathrm{~h}$ of culturing the retrieved oocytes in IVF-30 medium (G-IVF, 10135, Vitro-life, Sweden) supplemented with $10 \%$ synthetic serum substitute (Irvine Scientific, Santa Ana, CA); fertilization was confirmed by identification of pronuclei $16 \mathrm{~h}$ after insemination. All embryos were transferred into $\mathrm{G} 1$ medium $\left(\mathrm{G}^{-1}, 10,127\right.$, Vitro-life, Sweden) supplemented with $10 \%$ synthetic serum substitute and evaluated using the criteria of the Istanbul consensus [9]. For ICSI, sperm injection was performed according to the standard ICSI protocol, and fertilization was confirmed by identification of pronuclei $16 \mathrm{~h}$ after insemination. All embryos were transferred into G1 medium supplemented with $10 \%$ synthetic serum substitute and were evaluated using the criteria of the Istanbul consensus [9]. Day 4 to day 6 stage embryos were cultured in G2 medium (G-2, 10,131, Vitro-life, Sweden) supplemented with $10 \%$ synthetic serum substitute and embryos were evaluated using the criteria of the Istanbul consensus [10] .

Determination and monitoring of pregnancy: After day 3 fresh cleavage stage embryos transfer, blood or urine human chorionic gonadotropin (hCG) levels were measured at day 14 after embryos transfer. The patients with positive results underwent B-ultrasound examination 28 days later, and diagnosis criteria for pregnancy were the presence of gestational sac and primitive cardiac pulsation.

\section{Statistical analysis}

Statistical analysis was performed with the SPSS 23.0 software. An independent samples t-test was used to compare the baseline characteristics of the participants and the means of the number of oocytes recovered. The 
$\mathrm{X}^{2}$-test was used to compare $2 \mathrm{PN}$-fertilization, cleavage, day 3 good-quality embryo, blastulation, blastocyst utilization, day 5 blastocyst utilization, day 6 blastocyst utilization, embryo implantation and clinical pregnancy rates. Measurement data are mean \pm SD or \%. Statistical significance was defined at $P<0.05$.

\section{Results}

\section{Oocyte retrieval and embryo development outcomes}

The baseline characteristics of the total 1714 trial participants (Table 1) were similar in the oocyte retrieval difficulty group and the control group.

In Table 2, compared with the control group, the oocyte retrieval difficulty group had a significantly lower amounts of oocytes recovered, and a decreased 2PNfertilization rate as well as the decreased day 3 goodquality embryo, and blastocyst utilization (especially day 5 blastocyst utilization) rates.

\section{Oocyte retrieval and embryo development outcomes} between the two groups by different fertilization types

According to different fertilization types, the patients were further divided into IVF and ICSI subgroups. 2PNfertilization rate and embryo development potential were compared between the retrieval difficulty group and the control cases (Table 3).

In individuals receiving IVF, the difficulty group showed significantly less oocytes recovered and reduced rate of $2 \mathrm{PN}$-fertilization. The difficulty group had a day 3 good-quality embryo rate of $54.6 \%$ and a day 5 blastocyst utilization rate of $25.1 \%$, which were lower than the control group (56.0 and $29.2 \%$ respectively).

In cases receiving ICSI, the difficulty group showed significantly reduced amounts of MII stage oocytes, lower rates of day 3 good-quality embryo and blastocyst utilization rates (including day 5 and day 6).

\section{Clinical outcomes}

A total of 69 and 980 cases in the difficulty and control groups received fresh day 3 embryo transfer. Embryo transfer cycles / oocyte retrieval cycles in the difficulty group was $51.88 \%$ which was lower than the control (61.99\%), $P=0.026$; the average number of transfered embryos were $1.80 \pm 0.41$ and $1.82 \pm 0.38$, respectively. The rate of embryo implantation in the difficulty group was $53.2 \%$, which was lower than that of controls (58.7\%), although the difference was not significant. The clinical pregnancy, live birth and the early miscarriage rates between the two groups had no statistical significance. (Table 4).

\section{Neonatal outcomes}

Neonatal outcomes are presented in Table 5. Fifty nine and 905 babies were born from oocyte retrieval difficulty group and the control group. The median gestational ages were 38.22 and 37.62 weeks. Preterm birth (32-37 weeks) occurred in 25.4 and $27.3 \%$ of the oocyte retrieval difficulty group and the control group $(\mathrm{P}>0.05)$. The median birthweight of babies were $2934.48 \mathrm{~g}$ and $2851.99 \mathrm{~g}$ of the two groups. No significant difference was found for the rate of birthweight $(\mathrm{g})<2500$ of the live birth between the two groups.

\section{Discussion}

In vitro fertilization and embryo transfer, refers to the procedure that stimulates follicular development within the natural period or by gonadotropin administration, and then retrieves the mature ovum from the ovary for in vitro embryo culture and embryo transplantation

Table 1 Characteristics of the Participants at Baseline

\begin{tabular}{|c|c|c|c|}
\hline & $\begin{array}{l}\text { Difficulty group } \\
(N=133)\end{array}$ & $\begin{array}{l}\text { Control } \\
(N=1581)\end{array}$ & $P$ Value \\
\hline Age---yr & $28.99 \pm 3.14$ & $28.98 \pm 3.10$ & 0.99 \\
\hline Body-mass index & $20.82 \pm 1.32$ & $20.89 \pm 1.20$ & 0.52 \\
\hline Antral Follicle Count (AFC) & $17.50 \pm 6.18$ & $16.81 \pm 5.64$ & 0.50 \\
\hline Duration of infertility (year) & $3.56 \pm 2.51$ & $3.44 \pm 2.67$ & 0.79 \\
\hline \multicolumn{4}{|l|}{ Type of infertility } \\
\hline Primary & 81 & 980 & \\
\hline Secondary & 52 & 601 & \\
\hline \multicolumn{4}{|l|}{ Laboratory tests } \\
\hline Follicle-stimulating hormone---IU/liter & $7.66 \pm 3.10$ & $7.81 \pm 2.74$ & 0.67 \\
\hline Luteinizing hormone---IU/liter & $6.79 \pm 4.87$ & $6.14 \pm 3.98$ & 0.24 \\
\hline Estradiol---pg/ml & $48.45 \pm 36.88$ & $45.20 \pm 77.68$ & 0.68 \\
\hline Total testosterone---ng/ml & $1.30 \pm 5.88$ & $1.48 \pm 6.74$ & 0.83 \\
\hline
\end{tabular}


Table 2 Comparison of clinical parameters between the oocyte retrieval difficulty group and the control group

\begin{tabular}{|c|c|c|c|}
\hline & Difficulty group & Control & $P$ Value \\
\hline No. of cycles & 133 & 1581 & \\
\hline Total gonadotrophins dose & $1748.39 \pm 654.12$ & $1821.57 \pm 785.23$ & 0.79 \\
\hline E2 $(\mathrm{pg} / \mathrm{ml})$ in hCG Day & $3444.23 \pm 2224.30$ & $3451.94 \pm 1663.19$ & 0.99 \\
\hline Progesterone $(\mathrm{ng} / \mathrm{ml})$ in hCG Day & $0.95 \pm 0.79$ & $0.89 \pm 0.55$ & 0.59 \\
\hline Duration of $\mathrm{COH}$ & $10.66 \pm 3.14$ & $9.98 \pm 2.47$ & 0.149 \\
\hline No. of follicles aspirated & $12.17 \pm 4.92$ & $12.46 \pm 4.53$ & \\
\hline No. of recovered oocytes & $9.08 \pm 4.65$ & $12.13 \pm 5.27$ & $P<0.001$ \\
\hline 2PN-fertilization rate & $800 / 1116(71.7 \%)$ & $13,290 / 17565(75.7 \%)$ & 0.003 \\
\hline Cleavage rate & $774 / 800(96.8 \%)$ & $12,864 / 13290(96.8 \%)$ & 0.93 \\
\hline Day 3 good-quality embryo rate & $408 / 774(52.7 \%)$ & $7271 / 12864(56.5 \%)$ & 0.04 \\
\hline Blastulation rate & 203/423 (48.0\%) & $4050 / 7664(52.8 \%)$ & 0.06 \\
\hline Blastocyst utilization rate & 135/423 (31.9\%) & 2838/7664 (37.0\%) & 0.03 \\
\hline Day 5 blastocyst utilization rate & $90 / 423(21.3 \%)$ & 2004/7664 (26.1\%) & 0.02 \\
\hline Day 6 blastocyst utilization rate & $45 / 333(13.5 \%)$ & $834 / 5660(14.7 \%)$ & 0.58 \\
\hline
\end{tabular}

Note: Values are mean $( \pm$ SD) unless otherwise indicated

Table 3 Comparison of clinical parameters between the oocyte retrieval difficulty group and the control group by IVF or ICSI

\begin{tabular}{|c|c|c|c|}
\hline & Difficulty group & Control & $P$ Value \\
\hline \multicolumn{4}{|l|}{ Insemination methods } \\
\hline \multicolumn{4}{|l|}{ IVF } \\
\hline No. of cycles & 98 & 1180 & \\
\hline Age---yr & $29 \pm 3.06$ & $29 \pm 3.06$ & 0.99 \\
\hline No. of retrieved oocytes & $9.49 \pm 4.70$ & $12.08 \pm 5.29$ & $P<0.001$ \\
\hline 2PN-fertilization rate & 655/930 (70.4\%) & $10,584 / 14258(74.2 \%)$ & 0.01 \\
\hline Day3 good-quality embryo rate & $346 / 634(54.6 \%)$ & $5740 / 10245(56.0 \%)$ & 0.48 \\
\hline Blastulation rate & $177 / 342(51.8 \%)$ & $3269 / 5907$ (55.3\%) & 0.20 \\
\hline Blastocyst utilization rate & $122 / 342(35.7 \%)$ & $2297 / 5907$ (38.9\%) & 0.25 \\
\hline Day5 blastocyst utilization rate & 86/342 (25.1\%) & $1725 / 5907$ (29.2\%) & 0.11 \\
\hline Day 6 blastocyst utilization rate & $36 / 256(14.1 \%)$ & $572 / 4182(13.7 \%)$ & 0.85 \\
\hline \multicolumn{4}{|l|}{ ICSI } \\
\hline No. of cycles & 35 & 401 & \\
\hline Age---yr & $28.94 \pm 3.40$ & $28.90 \pm 3.20$ & 0.93 \\
\hline No. of retrieved oocytes & $7.94 \pm 4.35$ & $12.25 \pm 5.20$ & $P<0.001$ \\
\hline No. of MIl stage oocytes & $5.31 \pm 4.34$ & $8.25 \pm 5.14$ & 0.001 \\
\hline 2PN-fertilization rate & 145/186 (78\%) & 2706/3307 (81.8\%) & 0.19 \\
\hline Day 3 good-quality embryo rate & $62 / 140(44.3 \%)$ & $1531 / 2619(58.5 \%)$ & 0.001 \\
\hline Blastulation rate & $26 / 81(32.1 \%)$ & $781 / 1757(44.5 \%)$ & 0.03 \\
\hline Blastocyst utilization rate & 13/81 (16.0\%) & $541 / 1757(30.8 \%)$ & 0.005 \\
\hline Day 5 blastocyst utilization rate & $4 / 81(4.9 \%)$ & 279/1757 (15.9\%) & 0.004 \\
\hline Day6 blastocyst utilization rate & 9/77 (11.7\%) & $262 / 1216(21.5 \%)$ & 0.043 \\
\hline
\end{tabular}


Table 4 Clinical outcomes between the oocyte retrieval difficulty group and the control group

\begin{tabular}{|c|c|c|c|}
\hline & Difficulty group & control & $P$ Value \\
\hline Embryo transfer cycles & 69 & 980 & \\
\hline Fresh embryo transfer cycles/oocyte retrieval cycles & $69 / 133$ & $980 / 1581$ & 0.026 \\
\hline (\%) & $(51.88 \%)$ & $(61.99 \%)$ & \\
\hline Cycles having extra embryo cryopreservation & $53 / 69$ & $941 / 980$ & 0.00 \\
\hline (\%) & $(76.81 \%)$ & $(96.02 \%)$ & \\
\hline Mean embryos transferred & $1.80 \pm 0.41$ & $1.82 \pm 0.38$ & \\
\hline Endometrial thickness $(\mathrm{mm})$ in ET day & $9.80 \pm 1.70$ & $9.81 \pm 1.53$ & 0.959 \\
\hline Embryo implantation rate & $66 / 124(53.2 \%)$ & 1046/1782(58.7\%) & 0232 \\
\hline Pregnancy rate & $52 / 69(75.4 \%)$ & $715 / 980(73.0 \%)$ & 0.663 \\
\hline Spontaneous miscarriages & 3 & 29 & \\
\hline (\% per pregnancy, before 12 weeks) & $(5.8)$ & $(4.1)$ & 0.551 \\
\hline Spontaneous miscarriages & 3 & 16 & \\
\hline (\% per pregnancy, after 12 weeks) & $(5.8)$ & $(2.2)$ & 0.114 \\
\hline live birth rate & 46/69(66.7\%) & 670/980(68.4\%) & 0.769 \\
\hline
\end{tabular}

Note: Values are mean $( \pm$ SD) unless otherwise indicated

[11-14]. The first step of IVF-ET is to aspirate the matured oocytes from the ovary $[15,16]$. However, some patients may encounter difficulty on the day of oocyte collection. Among them, some may show failed oocyte retrieval, with consequently no embryo for transplantation [17]. Others, after repeated flushing, could obtain oocytes but a scant number of embryos suitable for transplantation [8]. This study retrospectively reviewed 133 cases who experienced difficulty on the day of oocyte collection, assessed the number of oocytes retrievaled, embryo development, clinical and neonatal outcomes.

It is generally admitted that the ovum can only worsen after in vitro handling $[18,19]$. Many factors, such as air quality, light, $\mathrm{pH}$, including in vitro operation, can cause a decrease in oocytes quality. The time taken for oocytes recovery and the difficulty for ovum retrieval are also important factors affecting oocytes quality [20, 21].

The current study included patients who experienced retrieval difficulty on the day of oocyte collection in 2017, alongside those with successful oocyte retrieval (control group), compared embryo development, the outcome of clinical pregnancy and neonatal outcomes between the two groups. We also compared embryo development in patients with different methods of fertilization, to analyze if the process of oocyte recovery might influence oocytes quality and embryo development potential. In cases receiving IVF, the difficulty group showed reduced rates of 2PN fertilization and day 5 blastocyst utilization compared with controls. In cases receiving ICSI, the difficulty group showed decreased number of MII stage oocytes, lower rates of day 3 goodquality embryo and day 5 blastocyst utilization.

In in vitro fertilization, good-quality embryos are important to successful embryo implantation [22, 23]. So, increasing the amount of retrieved oocytes is an effective way to improve the count of high-quality embryos [17, 23-25]. During ovum collection, some patients, due to various reasons, may experience retrieval difficulty, and finally obtain oocytes after repeated flushing. However, the repeated flushing pressure might cause early rupture of the follicular wall, which results in oocyte damage. Sometimes, oocyte damage could not be observed via the cumulus oocyte complexes, although it has already caused certain functional changes to the oocytes [26]. As reported previously, increased pressure during ovum retrieval would cause parthenogenetic activation in the oocyte, suggesting that pressure could

Table 5 Neonatal outcomes between the oocyte retrieval difficulty group and the control group

\begin{tabular}{llll}
\hline & Difficulty group & control & $P$ Value \\
\hline Pregnancies & 59 & 905 \\
Gestational age (weeks) & $38.22 \pm 3.54$ & $37.62 \pm 2.83$ \\
Gestational age (weeks) $<37 \mathrm{~W}$ & $15(25.4 \%)$ & $247(27.3 \%)$ & 0.755 \\
Birthweight (g) & $2934.48 \pm 825.81$ & $2851.99 \pm 625.79$ \\
Birthweight $(\mathrm{g})<2500$ & $12(20.3 \%)$ & $222(24.5 \%)$ & 0.467 \\
\hline
\end{tabular}

Note: Values are mean $( \pm \mathrm{SD})$ unless otherwise indicated 
change the physiological functions of the ovum [2]. In addition, repeated pressure on the oocytes could promote the formation of sterile oocytes, but the cumulus cells are critical for in vitro maturation, particularly cytoplasm maturation in oocytes [27]. In spite of nuclear maturation, oocytes without cumulus cells have limited developmental potential after fertilization, because cytoplasm maturation has been affected significantly. These embryos hardly develop into blastocysts, which yields very low clinical pregnancy rate $[18,28]$. On the other hand, oocytes retrieval difficulty may indicate that the patient might have more abnormal oocytes than the control group, some abnormal oocytes may reflect genetic abnormalities and the development potential of these oocytes were low [29].

As shown in this article, the difficulty group had significantly reduced oocyte utilization rate compared with controls. The retrieval difficulty group showed lower embryo implantation, cycles having embryos to transfer and clinical pregnancy rates compared with controls.

It would be good to know the cumulative delivery rate across all transfers per initiated cycle to evaluate the overall quality of the oocytes retrieved, but not all the patients having their frozen embryo transfer until now. So, we did not show this result in this study.

All in all, in patients having retrieval difficulty, how to improve the number of oocytes collected and especially improve the quality of the oocytes remains a challenge worth more widespread investigation.

\section{Conclusion}

In assisted reproduction, high-quality ovum is the basic and primary requirement for successful IVF [25]. Increasing the number of retrieved oocytes is an effective way to improve the number of transplantable or even high-quality embryos. Repeated flushing and extended time required for oocyte recovery during the process of oocyte retrieval, significantly reduced oocyte and embryo development potential.

\section{Abbreviations}

2PN: two pronuclei; hCG: human chorionic gonadotropin

ICSI: Intracytoplasmic sperm injection; IVF: In vitro fertilization; IVF-ET: In vitro fertilization and embryo transplantation

\section{Acknowledgements}

Not applicable.

\section{Authors' contributions}

L-hZ: Conception and design of study, drafting the manuscript. X-bN: AnaIysis and / or interpretation of data. FL: Analysis and / or interpretation of data. Z-pX: Analysis and / or interpretation of data. J-sF: Acquisition of data. $\mathrm{N}-\mathrm{yZ}$ : Conception and design of study, acquisition of data, revising the manuscript critically for important intellectual content. All authors read and approved the final manuscript.

\section{Funding}

Not applicable.

\section{Availability of data and materials}

The datasets used and/or analysed during the current study are available from the corresponding author on reasonable request.

\section{Ethics approval and consent to participate}

Our study protocol was approved by the Medical Ethics Committee of Drum Tower Hospital Affiliated with Nanjing University Medical College. All patients admitted at our center had consented to the anonymous use of their medical data for scientific research, publication and/or quality assessment, and informed consents were previously signed at the beginning of their treatments. Because the study was designed to retrospectively analyze the data of patients who had finished their treatments, further consent was not necessary.

\section{Consent for publication}

Not applicable.

\section{Competing interests}

The authors declare that they have no competing interests.

Received: 21 August 2019 Accepted: 5 November 2019

Published online: 05 December 2019

\section{References}

1. Baber R, Porter R, Picker R, Robertson R, Dawson E, Saunders D. Transvaginal ultrasound directed oocyte collection for in vitro fertilization: successes and complications. J Ultrasound Med. 1988;7(7):377-9.

2. Tan SL, Waterstone J, Wren M, Parsons J. A prospective randomized study comparing aspiration only with aspiration and flushing for transvaginal ultrasound-directed oocyte recovery. Fertil Steril. 1992;58(2):356-60.

3. Brinsmead M, Stanger J, Oliver M, Shumack J, Raymond S, Clark L. A randomized trial of laparoscopy and transvaginal ultrasound-directed oocyte pickup for in vitro fertilization. J In Vitro Fert Embryo Transf. 1989; 6(3):149-54

4. Lattes $K$, Checa MA, Vassena $R$, Brassesco M, Vernaeve $V$. There is no evidence that the time from egg retrieval to embryo transfer affects live birth rates in a freeze-all strategy. Hum Reprod. 2017;32(2):368-74.

5. van der Gaast MH, Eijkemans MJ, van der Net JB, de Boer EJ, Burger CW, van Leeuwen FE, et al. Optimum number of oocytes for a successful first IVF treatment cycle. Reprod Biomed Online. 2006;13(4):476-80.

6. Sunkara SK, Rittenberg V, Raine-Fenning N, Bhattacharya S, Zamora J, Coomarasamy A. Association between the number of eggs and live birth in IVF treatment: an analysis of 400135 treatment cycles. Hum Reprod. 2011; 26(7):1768-74

7. El HE, Balen AH, Tan SL. A prospective study comparing the outcome of oocytes retrieved in the aspirate with those retrieved in the flush during transvaginal ultrasound directed oocyte recovery for in-vitro fertilization. $\mathrm{Br} J$ Obstet Gynaecol. 1992:99(10):841-4.

8. Waterstone JJ, Parsons $\mathrm{JH}$. A prospective study to investigate the value of flushing follicles during transvaginal ultrasound-directed follicle aspiration. Fertil Steril. 1992;57(1):221-3.

9. Prados FJ, Debrock S, Lemmen JG, Agerholm I. The cleavage stage embryo. Hum Reprod. 2012;27(Suppl 1):i50-71.

10. Hardarson T, Van Landuyt L, Jones G. The blastocyst. Hum Reprod. 2012; 27(Suppl 1):i72-91.

11. Ho JR, Paulson RJ. Modified natural cycle in in vitro fertilization. Fertil Steril. 2017:108(4):572-6.

12. Simoni MK, Mu L, Collins SC. Women's career priority is associated with attitudes towards family planning and ethical acceptance of reproductive technologies. Hum Reprod. 2017;32(10):2069-75.

13. Chambers GM, Paul RC, Harris K, Fitzgerald O, Boothroyd CV, Rombauts $L$, et al. Assisted reproductive technology in Australia and New Zealand: cumulative live birth rates as measures of success. Med J Aust. 2017; 207(3):114-8

14. Haas J, Zilberberg E, Dar S, Kedem A, Machtinger R, Orvieto R. Coadministration of $\mathrm{GnRH}$-agonist and hCG for final oocyte maturation (double trigger) in patients with low number of oocytes retrieved per number of preovulatory follicles--a preliminary report. J Ovarian Res. 2014;7:77.

15. Awonuga A, Waterstone J, Oyesanya O, Curson R, Nargund G, Parsons J. A prospective randomized study comparing needles of different diameters for 
transvaginal ultrasound-directed follicle aspiration. Fertil Steril. 1996;65(1): 109-13.

16. Stanger JD, Yovich JL. Follicle recruitment determines IVF productivity rate via the number of embryos frozen and subsequent transfers. Reprod Biomed Online. 2013;27(3):286-96.

17. Nakamura M, Yamashita Y, Hayashi A, Saito N, Yu M, Hayashi M, et al. Analyzing the risk factors for a diminished oocyte retrieval rate under controlled ovarian stimulation. Reprod Med Biol. 2017;16(1):40-4.

18. Palmsten K, Homer MV, Zhang Y, Crawford S, Kirby RS, Copeland G, et al. In vitro fertilization, interpregnancy interval, and risk of adverse perinatal outcomes. Fertil Steril. 2018;109(5):840-8.

19. James E, Jenkins TG. Epigenetics, infertility, and cancer: future directions. Fertil Steril. 2018;109(1):27-32.

20. Calhaz-Jorge C, De Geyter C, Kupka MS, de Mouzon J, Erb K, Mocanu E, et al. Assisted reproductive technology in Europe, 2013: results generated from European registers by ESHRE. Hum Reprod. 2017;32(10):1957-73.

21. Cai $Q$, Wan F, Huang $K$, Zhang $H$. Does the number of oocytes retrieved influence pregnancy after fresh embryo transfer? PLOS ONE. 2013;8(2):e56189.

22. Huo X, Chen D, He Y, Zhu W, Zhou W, Zhang J. Bisphenol-A and Female Infertility: A Possible Role of Gene-Environment Interactions. Int J Environ Res Public Health. 2015;12(9):11101-16.

23. Ji J, Liu Y, Tong XH, Luo L, Ma J, Chen Z. The optimum number of oocytes in IVF treatment: an analysis of 2455 cycles in China. Hum Reprod. 2013; 28(10):2728-34.

24. Magnusson A, Kallen K, Thurin-Kjellberg A, Bergh C. The number of oocytes retrieved during IVF: a balance between efficacy and safety. Hum Reprod. 2018:33(1):58-64.

25. Steward RG, Lan L, Shah AA, Yeh JS, Price TM, Goldfarb JM, et al. Oocyte number as a predictor for ovarian hyperstimulation syndrome and live birth: an analysis of 256,381 in vitro fertilization cycles. Fertil Steril. 2014;101(4):967-73.

26. Pelinck MJ, Hoek A, Simons AH, Heineman MJ, van Echten-Arends J, Arts EG. Embryo quality and impact of specific embryo characteristics on ongoing implantation in unselected embryos derived from modified natural cycle in vitro fertilization. Fertil Steril. 2010;94(2):527-34.

27. Artini PG, Tatone C, Sperduti S, D'Aurora M, Franchi S, Di Emidio G, et al. Cumulus cells surrounding oocytes with high developmental competence exhibit down-regulation of phosphoinositol 1,3 kinase/protein kinase B (PI3K NAKT) signalling genes involved in proliferation and survival. Hum Reprod. 2017;32(12):2474-84.

28. Ko DS, Lee SH, Park DW, Yang KM, Lim CK. Pregnancy and fertilization potential of immature oocytes retrieved in intracytoplasmic sperm injection cycles. Clin Exp Reprod Med. 2015;42(3):118-25.

29. Rienzi L, Balaban B, Ebner T, Mandelbaum J. The oocyte. Hum Reprod. 2012; 27(S1):i2 -i21, 2012.

\section{Publisher's Note}

Springer Nature remains neutral with regard to jurisdictional claims in published maps and institutional affiliations.

Ready to submit your research? Choose BMC and benefit from:

- fast, convenient online submission

- thorough peer review by experienced researchers in your field

- rapid publication on acceptance

- support for research data, including large and complex data types

- gold Open Access which fosters wider collaboration and increased citations

- maximum visibility for your research: over $100 \mathrm{M}$ website views per year

At BMC, research is always in progress.

Learn more biomedcentral.com/submissions 